Environmental Factors", and "Some Quantitative Aspects of Translocation". Arrangement under such headings has inevitably led to repetition; for example, work on phloem exudation, the effects of temperature and water stress, the role of sources and sinks, and constituents of the translocation stream are each dealt with in more than one part of the book. The various sections are really separate essays. Perhaps they represent individual contributions from the two authors.

The book nonetheless bears the unmistakable stamp of Professor Crafts; over the years he has been an unremitting advocate of Münch's pressure flow hypothesis, and this is evident all through the book. In a sentence thie views of the authors are: the sieve plate pores are "open" and freely permit a mass flow which is impelled by a gradient of turgor pressure in the sieve tubes between the "source" and "sink" regions of the plant. Every fact is brought to the bar of this concept and judged as to its consistency with it. The authors evince no shadow of doubt that their views are right and this makes the book all the more stimulating. But many other physiologists and cytologists are less sure.

P. E. Weatherley

\section{Invertebrate Structure}

Textbook of Zoology: Invertebrates. Edited by A. J. Marshall and W. D. Williams. Seventh edition of $A$ Textbook of Zoology. Vol. 1. By the late T. Jeffrey Parker and the late William A. Haswell. Pp. xix +874 . (Macmillan: London and Basingstoke, May 1972.) $£ 5.70$.

THIRTY-Two years have elapsed since the last (sixth) edition of Parker and Haswell's well-known textbook appeared of which the first edition was published in 1898. This new edition has been edited by the late Professor A. J. Marshall and by Dr Williams with the cooperation of several distinguished authorities on different groups of invertebrates. When the sixth edition was new, its rival was The Invertebrata by Borradaile, Eastham, Potts and Saunders, popularly known as "BEPS", and I for one, as a student, much preferred it to "Parker and Haswell". The scene has now changed considerably and this new edition may be compared with the textbooks of Barnes and of Meglitsch. These are written in American of course, and it is pleasant to have once more a new textbook-for this is really what it is, in spite of its historical associations-written in English. There is little doubt that "Marshall and Williams" will win support and the choice between these three excellent textbooks will clearly be dictated by the type and extent of the course being run. All are specially for undergraduate use to supplement lectures and practicals and to provide an introduction to invertebrate biology by instilling a working knowledge of invertebrate structure and taxonomy.

Several specialists have contributed sections dealing with certain groups. I was particularly pleased to see that the Protozoa have been contributed by Eugene Kozloff, the Platyhelminthes and nemertines by J. Llewellyn, the Cnidaria and ctenophores by Cadet Hand, Bryozoa by J. S. Ryland and the echinoderms by Barraclough Fell. This should be enough to reassure the reader that this is not just a last attempt to prop up an aged text but rather a genuine effort to give to it a new lease of life. Clearly the larger phyla have more devotees, and to single out the chapters mentioned is not to cast aspersions on other sections. How delighted and perhaps amazed Parker and Haswell would have been to see this new edition illustrated with stereoscan photographs of Ascaris lips!

Marshall and Williams's book is well arranged and the figures well reproduced in filmset with half-tones and line drawings alongside. It continues the Parker and Haswell tradition of providing a large number of illustrations (there are 803 ) to give as wide a range of invertebrates as possible. Some of these illustrations are the same as in earlier editions but many of them are new. The book continues the "type" approach: there is much to recommend this for an elementary book which is designed as a basis for further courses on invertebrate biology or physiology. Indeed this is the strength or weakness - according to one's point of viewof the present text. There is very little biology and much morphology. Many students today seem to find courses designed to provide a working knowledge of the invertebrates irksome, if not dull, and the success of this book will clearly depend on how it is used. Taken alone it is somewhat oldfashioned. But it could be an ideal companion for a lecture course in which the biology and physiology were stressed, for one would complement the other. I personally believe that the acquisition of a working knowledge of animal structure and taxonomy is an essential prerequisite for further work, however specialized this may be. Whether this purpose is served best by Marshall and Williams or by one or other of the other textbooks currently available depends on the teachers concerned, but it is quite clear that this new edition will take its place among the two or three in English (and there are only two or three) worth considering.

There are some details with which $I$ would personally disagree: the prominence given to Neopilina, for example, is, I think, unjustified in the present state of our knowledge. The position is correctly stated, but the relative sizes of the figures devoted to Neopilina and the "archetypal mollusc" should surely have been reversed. Some other figures are small, but they are at least clear. While most are adequately labelled some are not and this applies particularly to many of the drawings of Crustacea. More serious, I think, is the prominence given to Sharov's theory of the origin of the arthropod. While Dr S. M. Manton subjected Sharov's views concerning the specialized polychaete Spinther to the derision it deserved, this is not mentioned, and for students to equate this theory with those expressed by Tiegs and Manton is in my view misleading.

The book excludes the protochordates and in this follows the tradition of Parker and Haswell. No doubt they will be included in volume 2 together with the vertebrates. The insects and the "minor groups"-in their commonly accepted sense-are included.

On the whole the reappearance of this book provides an agreeable experience: a reassurance that many others still regard the acquisition of an appreciation of morphological detail as an essential discipline for any student of zoology. This is a work which most of us who teach will want on our shelves to join the others to recommend to students at different stages of their study. 'This is a "primer" (for all its faults) for Barrington's book, and is essential for all zoological libraries.

R. P. Dales

\section{Likelihood}

Likelihood: An Account of the Statistical Concept of Likelihood and its Applications to Scientific Inference. By A. W. F. Edwards. Pp. xv+235. (Cambridge University: London, March 1972.) £3.80; $\$ 12.50$.

How are conclusions reasonably drawn from research data? The pertinence of the question is indicated by the fact that three essentially different answers are represented in the recent research literature of genetics: suppose the probability of data $D$, on hypothesis $H$, is given by the known function $P(D \mid H)$. The likelihood approach to data analysis is based exclusively on the likelihood function $P\left(D^{*} \mid H\right)$ where $D^{*}$ is the data observed. The likelihood ratio $P\left(D^{*} \mid H^{\prime}\right) / P\left(D^{*} \mid H^{\prime \prime}\right)$ is interpreted as characterizing numerically the support by data $D^{*}$ for hypothesis $H^{\prime}$ as against $H^{\prime \prime}$. Bayesian interpretations are based exclusively on $P(H) P\left(D^{*} \mid H\right)$, where $P(H)$ is - the long-controversial prior 